\title{
Increased face detection responses on the mooney faces test in people at clinical high risk for psychosis
}

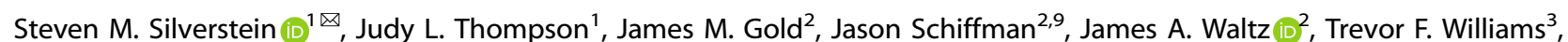
Richard E. Zinbarg ${ }^{3}$, Vijay A. Mittal (iD) ${ }^{3}$, Lauren M. Ellman ${ }^{4}$, Gregory P. Strauss ${ }^{5}$, Elaine F. Walker ${ }^{6}$, Scott W. Woods ${ }^{7}$, Jason A. Levin (iD ${ }^{5}$, Eren Kafadar ${ }^{7}$, Joshua Kenney ${ }^{7}$, Dillon Smith ${ }^{8}$, Albert R. Powers ${ }^{7,10}$ and Philip R. Corlett (DD $^{7,10}$

Identifying state-sensitive measures of perceptual and cognitive processes implicated in psychosis may allow for objective, earlier, and better monitoring of changes in mental status that are predictive of an impending psychotic episode, relative to traditional selfreport-based clinical measures. To determine whether a measure of visual perception that has demonstrated sensitivity to the clinical state of schizophrenia in multiple prior studies is sensitive to features of the at-risk mental state, we examined differences between young people identified as being at clinical high risk for psychosis (CHR; $n=37)$ and non-psychiatric matched controls $(n=29)$ on the Mooney Faces Test (MFT). On each trial of the MFT, participants report whether they perceive a face in a degraded face image. The CHR group reported perceiving a greater number of faces in both upright and inverted MFT stimuli. Consistent with prior work, males reported more faces on the MFT than females in both conditions. However, the finding of greater reported face perception among CHR subjects was robustly observed in the female CHR group relative to the female control group. Among male CHR participants, greater reported face perception was related to increased perceptual abnormalities. These preliminary results are consistent with a small but growing literature suggesting that heightened perceptual sensitivity may characterize individuals at increased clinical risk for psychosis. Further studies are needed to determine the contributions of specific perceptual, cognitive, and motivational mechanisms to the findings.

npj Schizophrenia (2021)7:26; https://doi.org/10.1038/s41537-021-00156-1

\section{INTRODUCTION}

The clinical high risk (CHR) approach to studying psychosis has led to advances in the ability to reliably identify and effectively treat those most likely to develop a psychotic disorder ${ }^{1,2}$. Most CHR approaches use data from clinical interviews to define at-risk subgroups and to predict conversion to a psychotic disorder; however, this approach is less than optimal in several important respects ${ }^{3}$. One potential way to improve clinical utility is to incorporate performance-based measures of specific neurocomputational processes that are linked to one or more aspects of schizophrenia. However, when CHR initiatives have incorporated performance-based assessments, they have often been traitlinked measures that are unlikely to be sensitive to, or predictive of, changes in mental status ${ }^{4}$. The incorporation of state-sensitive performance-based measures with established links to the pathophysiological mechanisms implicated in symptom genesis may allow for more objective and effective monitoring of changes in mental status that are predictive of an impending psychotic episode or a positive response to treatment ${ }^{4,5}$. While this approach to psychosis risk assessment is just emerging, there are already multiple studies of schizophrenia documenting that relatively brief and non-invasive behavioral and electrophysiological measures can tap into mechanisms thought to be involved in positive $^{6-8}$, negative ${ }^{9-12}$, disorganized ${ }^{13-15}$ or motor ${ }^{16}$ symptoms, and that these measures are sensitive to changes in the severity level of the symptom cluster with which they are correlated ${ }^{14,17,18}$.

To inform the identification of measures that are sensitive to processes underlying symptom development and expression in the at-risk mental state, we investigated the performance of individuals at $\mathrm{CHR}$ on a well-validated assessment of visual perception, the Mooney Faces Test $\left(\mathrm{MFT}^{19}\right)$. In its most basic form, participants view images of faces that have been transformed to two-tone (thresholded, or black and white only) images (Fig. 1). The images are presented one at a time, and, on each trial, subjects report whether they perceive a face in the image. Identifying faces in MFT images relies heavily on the ability to perceptually organize the black and white segments into a holistic representation (i.e., to achieve perceptual closure), culminating in face perception. The individual images are rendered such that there is a range of difficulty levels with regard to face detection across the images, from very easy to very difficult.

Because two-tone images do not lend themselves to volumetric interpretations (i.e., they are difficult to interpret as threedimensional), perception of faces in MFT images requires the influence of top-down representations of 2D faces that are stored in memory ${ }^{20}$. As such, face priors applied during performance of the MFT are thought to involve a combination of stored representations of $3 D$ (i.e., real) faces and $2 D$ face images ${ }^{21,22}$. When MFT stimuli are inverted, a face is usually not perceived. This is likely for two reasons: (1) the inverted images no longer lend themselves to grouping into a face gestalt ${ }^{23}$ (a conclusion supported by findings of significantly less fusiform face area activation in response to inverted relative to upright MFT images) $^{24}$; and (2) people do not have stored representations of inverted 2D face images that can be applied as templates in a top-

\footnotetext{
${ }^{1}$ University of Rochester Medical Center, New York, NY, USA. ${ }^{2}$ University of Maryland School of Medicine, Baltimore, MD, USA. ${ }^{3}$ Northwestern University, Evanston, IL, USA. ${ }^{4}$ Temple University, Philadelphia, PA, USA. ${ }^{5}$ University of Georgia, Athens, GA, USA. ${ }^{6}$ Emory University, Atlanta, GA, USA. ${ }^{7}$ Yale University, New Haven, CT, USA. ${ }^{8}$ Princeton University, Princeton, NJ, USA. ${ }^{9}$ Present address: University of California, Irvine, CA, USA. ${ }^{10}$ These authors contributed equally Albert R. Powers, Philip R. Corlett.

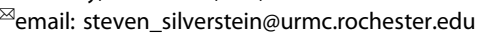



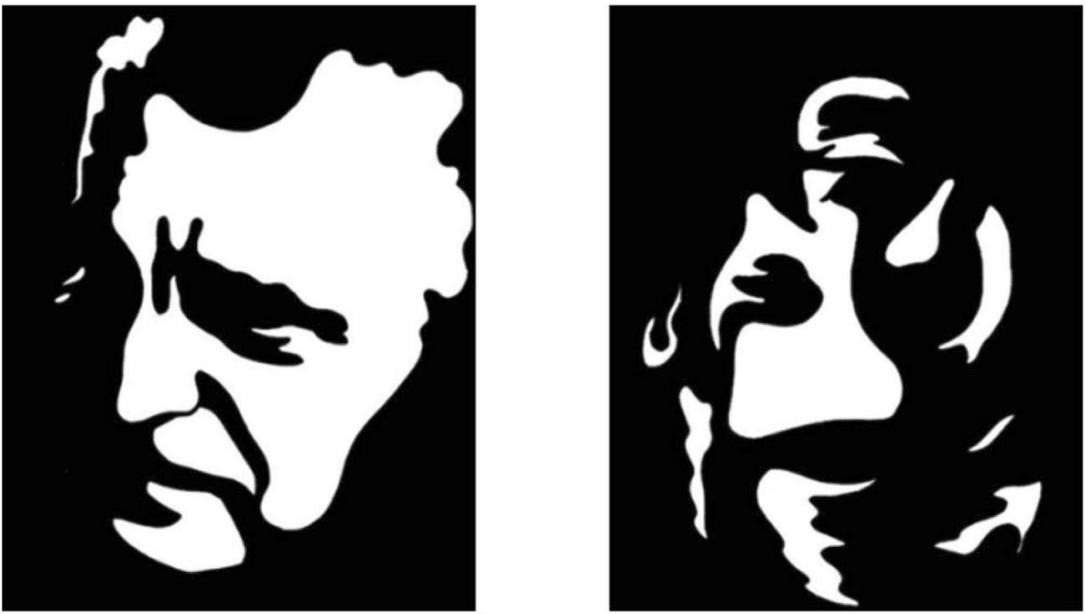

Fig. 1 Examples of Mooney Faces Test stimuli. Left - A mid-level difficulty stimulus from the upright condition; Right - a mid-level difficulty stimulus from the inverted condition.

down fashion unless they have undergone extensive training to efficiently process such images as faces.

Prior studies of the MFT in schizophrenia have reported impairments (operationalized as identification of fewer faces), consistent with the perceptual organization difficulties often observed in this population (reviewed $\mathrm{in}^{25,26}$ ). Such perceptual organization impairments are related to higher levels of disorganized symptoms in people with an established diagnosis and chronic course of schizophrenia ${ }^{13}$, and to reduced gamma power during task performance ${ }^{27}$. The relationships between these neuro-perceptual and behavioral alterations are consistent with the hypothesis that schizophrenia is characterized by widespread impairments in contextual modulation. This is defined as modifications in the grouping, timing, salience, or interpretation of stimulus-related neural signals, without changing the representation of basic stimulus features themselves. In this way, contextual modulation serves to optimize processing and adaptation across changing contexts ${ }^{28}$. An impairment in this canonical cortical algorithm has been hypothesized to be the basis of multiple failures of representation organization in schizophrenia (e.g., in auditory and visual perception, in thought and language $)^{28,29}$. These data suggest that alterations in MFT performance in a CHR group would be associated with severity of disorganized symptoms. In addition, due to the top-down nature of the processing involved, and the need to evaluate the stimuli in terms of 2D and 3D face priors, as discussed above, the task may also leverage the stored knowledge and updating processes associated with predictive coding. Findings from two recent magnetoencephalography (MEG) studies are consistent with the notion that predictive processes are engaged during the $\mathrm{MFT}^{30,31}$. A growing literature has linked alterations in predictive processing with positive symptoms in schizophrenia, ${ }^{5,32-34}$, suggesting that MFT performance among those at CHR for psychosis might be associated with severity of attenuated positive symptoms.

An open question was whether young people at CHR for psychosis would perceive an increased or decreased number of faces on the MFT relative to healthy controls. A decrease would be expected based on the multiple studies of reduced face perception on the MFT in participants with schizophrenia, as described above. However, there are several cases in which unmedicated first-episode patients, or high-risk subjects, evince heightened sensitivity on perceptual measures. For example, although it is well established that chronic schizophrenia is associated with impairments in the perception of low-contrast stimuli, regardless of medication status ${ }^{26}$, multiple studies ${ }^{26}$ have indicated enhanced contrast sensitivity in unmedicated first episode patients relative to controls. While no data exist on MFT performance among CHR samples, a study of perceptual organization in a sample of adult patients with schizotypal features and a history of brief, limited, intermittent psychotic symptoms (i.e., aspects of CHR status) observed enhanced performance in this high-risk group relative to controls ${ }^{35}$. In addition, a CHR study conducted by Teufel et al. that investigated whether prior exposure to original, unaltered images enhanced perception when viewing two-tone versions of those images (which were similar to MFT images) reported that CHR participants were superior to controls in utilizing that (experiment-provided) prior knowledge to recognize the content of the ambiguous twotone images ${ }^{36}$. A second study reported in that paper demonstrated that, in a non-clinical sample of participants who were considered prone to psychosis based on measures of schizotypy, enhanced use of priors was related to psychotic-like perceptual distortions but not to quasi-delusion-like experiences. However, a study of unmedicated first episode patients using the MFT found reduced face perception ${ }^{37}$. Therefore, we expected that $\mathrm{CHR}$ participants would differ from controls in their MFT performance, and that their performance would be related to severity of perceptual abnormalities and/or disorganized symptoms specifically, but we were agnostic at the outset regarding the direction of the hypothesized significant between-group difference. Of note, unlike in the Teufel et al. study described above, we did not provide subjects with prior exposure to the unaltered face images, as we sought to determine the 'naïve' MFT performance of subjects, unaffected by prior exposure to any form of the images.

\section{RESULTS}

\section{Demographic characteristics}

Sixty-six participants (37 CHR and 29 healthy controls $(\mathrm{HC})$ ) were included in this study. Thirty-five of these participants (21 CHR, 14 HC) were recruited at Northwestern University, and 31 (16 CHR, 15 $\mathrm{HC}$ ) at the University of Georgia (see Methods). Demographic and clinical characteristics are provided in Table 1. CHR and HC groups did not differ significantly on age $[t(64)=1.26, p=0.21]$, years of education $[t(62)=-0.41, p=0.68]$, or gender ratio $\left[x^{2}(1)=1.97\right.$, $p=0.16]$. Due to the small numbers in some of the race categories, the $\mathrm{CHR}$ and $\mathrm{HC}$ groups were compared on the proportion of Caucasian vs. non-Caucasian participants. The 
groups did not differ significantly in racial composition using this method of classification: $x^{2}(1)=0.93, p=0.34$.

\section{Between-condition differences in Mooney Faces Test performance}

As expected, the frequency of reporting faces was greater for the upright condition compared to the inverted condition $(73.36 \%$ vs. $33.34 \%$, respectively): $t(65)=16.75, p<001, d=2.24$.

\section{Between-group comparisons on Mooney Faces Test performance}

Compared to $\mathrm{HC}$ participants, the CHR group reported perceiving a significantly greater number of faces in the upright condition of the MFT $[t(64)=3.68, p<0.001$, Cohen's $d=0.89$; see Fig. 2a]. The CHR group also reported perceiving a greater number of faces in the inverted condition, although this difference was only at a trend level $[t(64)=1.95, p=0.055, d=0.49$; see Fig. $2 \mathrm{~b}]$. The degree to which CHR participants perceived a greater number of faces than HC participants was similar in the two conditions, as

\begin{tabular}{|lll|}
\hline Table 1. Demographic and clinical characteristics by group. \\
\hline Characteristic & $\mathrm{CHR}(n=37)$ & $\mathrm{HC}(n=29)$ \\
\hline Age & $21.57(2.47)$ & $20.83(2.25)$ \\
Gender, \% female & $59.5 \%$ & $75.9 \%$ \\
Participant education (years) & $14.44(2.01)$ & $14.64(1.81)$ \\
Race, \% & & \\
Caucasian & $56.8 \%$ & $44.8 \%$ \\
African American & $27.0 \%$ & $10.4 \%$ \\
Asian & $8.1 \%$ & $34.5 \%$ \\
Hispanic-Latino & $8.1 \%$ & $3.4 \%$ \\
Multiracial & $0.0 \%$ & $6.9 \%$ \\
SIPS & \multicolumn{2}{|c|}{} \\
Positive symptom subscale & $11.70(2.98)$ & - \\
P3 Grandiose ideas & $1.33(1.45)$ & - \\
P4 Perceptual abnormalities/hallucinations & $2.83(1.25)$ & - \\
P5 Disorganized communication & $1.47(1.16)$ & - \\
Negative symptom subscale & $6.73(5.76)$ & - \\
Disorganized symptom subscale & $4.41(2.51)$ & - \\
\hline
\end{tabular}

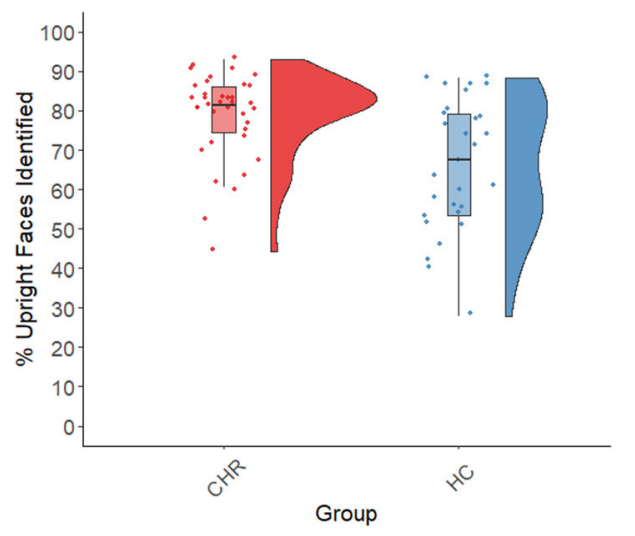

indicated by a non-significant group $\mathrm{x}$ condition interaction effect in a mixed-model ANOVA: $F(1,64)=0.43, p=0.51$.

In a univariate ANOVA conducted to assess gender differences, a significant main effect was observed for gender $[(F(1,62)=4.82$, $p=0.032, d=.57]$, indicating that, collapsed across groups and test conditions, males and females differed significantly on the number of faces reported. Specifically, males reported perceiving faces on $8.09 \%$ more trials than females in the upright condition, and $10.0 \%$ more trials in the inverted condition. The degree of greater face reporting among males was statistically similar across the two conditions as indicated by a non-significant gender $\mathrm{x}$ condition interaction term $[F(1,62)=0.62, p=0.44]$. Post-hoc $t$ tests indicated that the difference between males and females was significant in the upright condition $[t(64)=2.12, p=.038, d=$ $0.57]$ and at a trend level in the inverted condition $[t(64)=2.00$, $p=0.050, d=0.51]$. The overall pattern of findings, in terms of gender differences, was similar for the $\mathrm{CHR}$ and $\mathrm{HC}$ groups as indicated by a non-significant group $\times$ gender $\times$ condition interaction $[F(1,62)=0.103, p=0.79]$, although it must be noted that this analysis was underpowered. Female CHR subjects reported more faces than female $\mathrm{HC}$ subjects for upright faces [13.85\% increase; $t(42)=3.31, p=0.002, d=0.998]$, and the difference was at a trend level for inverted faces [9.18\% increase; $t(42)=1.68, p=0.10, d=.51]$. Among males, the difference was not significant for either the upright $[6.36 \%$ increase; $t(20)=1.08$, $p=0.29, d=0.42]$ or inverted [5.16\% increase; $t(20)=0.53, p=$ $0.60, d=0.23$ ] conditions. Although the findings were in the same direction as for females, the samples sizes in the male CHR and HC groups ( $n=15$ and 7, respectively) were smaller than the sizes for the corresponding female subgroups $(n=22$ and 22), thereby reducing power for these subgroup analyses.

\section{Correlations with psychosis-risk symptoms}

Among the CHR participants, there were no significant associations between the number of faces reported in either the upright or inverted condition and total scores on the SIPS positive, negative or disorganized symptom subscales, or with severity of any of the individual symptoms examined (i.e., perceptual abnormalities/hallucinations (P4), grandiosity (P3), or communication disturbance (P5); see Table 2). We also examined these correlations separately for male and female CHR participants, given the gender differences observed in task performance. Among these correlations, only one was significant: for male CHR subjects $(n=14)$, the number of faces reported in the upright condition was significantly associated with severity of perceptual abnormalities/hallucinations $(r=0.73, p=0.003$; see Table 2 and Supplemental Results for scatterplot). This correlation remained

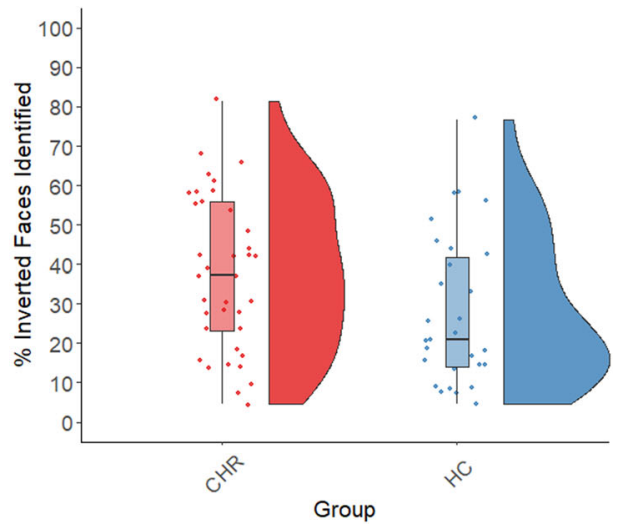

Fig. 2 Group differences and distributions of scores (\% of trials on which a face was reported) for the CHR and CON groups in the MFT conditions. Left: Box and raincloud plots showing the medians, inter-quartile ranges, and outliers for each group in the Upright Faces condition. The means were similar to the medians and were: $\mathrm{CHR}=78.88$ ( $\mathrm{SD}=10.86), \mathrm{CON}=66.32$ (SD $=16.75)$. Right: Corresponding data for the Inverted Faces condition. The means were similar to the medians and were: $\mathrm{CHR}=37.46(\mathrm{SD}=19.56), \mathrm{CON}=28.08(\mathrm{SD}=19.07)$. 
4 the same after Hadi outlier correction, and remained significant when calculated as a Spearman Rho $\left(r_{\mathrm{s}}=0.66, p<0.02\right)$. Nevertheless, given that this was the only significant correlation in this set of analyses, that the coefficient was unusually large, and that it was much larger for males than females (female $r=-0.05, p>.99$; male-female difference: $Z=2.59, p=0.01$ ), it is possible that this is a spurious finding rather than an indication of a link between heightened face perception on the MFT and severity of perceptual abnormalities among CHR participants. Thus, it should be viewed with caution.

\section{DISCUSSION}

Compared to controls, individuals at CHR reported seeing more faces on the MFT in both the upright and inverted conditions. These data imply that young people at clinical high risk for psychosis may have a heightened tendency to perceive faces in ambiguous visual stimuli that possess aspects of a facial gestalt. In a more general sense, this may reflect an increase in the signaling of social significance in situations in which information is minimal or absent. Similar to past studies, males reported perceiving a greater number of faces than females. However, the finding of greater reported face perception among CHR subjects was robustly observed among female CHR subjects relative to female controls.

This study had a number of important limitations. One is the small sample size, which is associated with low statistical power to detect small to medium effect sizes, and which could make the data unduly vulnerable to the impact of outliers, emphasizing the need for replication in a larger sample. This is the case even though we used several methods to examine and mitigate outlier effects, which we believe were minimal in this study. The sample size issue is especially relevant to the male vs. female comparisons between the CHR and control groups, as noted above. A second limitation is the lack of balance in gender composition between the CHR and HC groups. The CHR group was $41 \%$ male, and the $\mathrm{HC}$ group was $24 \%$ male. While this difference was not statistically significant, it is still possible that the difference could have affected group means on the MFT, given prior findings that perception of faces on the MFT may be more pronounced in males ${ }^{38-40}$, which is also what we observed. However, evidence that our findings are not due to a gender imbalance between groups is that although female subjects reported fewer faces than males overall, female CHR subjects reported significantly more faces in the upright condition than did HC females, and they reported more faces at a trend level in the inverted condition. A third limitation is the lack of a direct or strong indirect method for determining what subjects actually perceived, independently of what they reported perceiving. This leaves open the possibility that group differences in MFT performance could have been driven by the use of a more liberal decision criterion to indicate that a face was present among CHR participants. Unfortunately, all of our MFT stimuli contained face images (even if inverted) and thus there were no images that could truly be considered 'noise' stimuli, precluding a signal detection analysis to distinguish between perceptual and decisional contributions to the task. See Supplemental Discussion for a further discussion of issues involved in using signal detection theory, or generating psychometric functions based on stimulus difficulty level, using the original MFT stimulus set. In future studies, it would be possible to perform a signal detection analysis if either scrambled faces, or, preferably, 'Mooney objects' (which contain no face components) were used as catch stimuli. There are also newer versions of the MFT that have validated difficulty scores for a large number of items ${ }^{41}$, which would facilitate the generation of psychometric functions across stimuli for subjects and groups. For the stimulus set used in the present study, no such data are available (see Supplemental Discussion for more on this issue). 
The contributions of perceptual changes versus adoption of a more liberal criterion for responding that a face was seen could also be addressed by including additional psychophysiological or behavioral measures. For example, one could investigate fusiform face area (FFA) activation in response to upright vs. inverted Mooney face images. Previous $\mathrm{fMRI}$ studies indicate that activation of the FFA is significantly greater in response to upright relative to inverted $^{20,24}$ or scrambled ${ }^{42}$ faces. Similar findings have been observed using $\mathrm{MEG}^{27}$. Therefore, groups could be compared on the extent to which their reports of seeing faces correspond to FFA activation. An EEG study demonstrated that perception of upright faces was associated with a significant increase in synchronized gamma-band activity between parietal-occipital and fronto-temporal regions, and that this increase was not observed during viewing of inverted versions of the same images $^{43}$. Thus, differences in level of synchrony increase in response to MFT images may be helpful in classifying instances of face perception vs. reports driven by post-perceptual (decisional) factors. Future studies could also ask subjects to report what kind of face they perceive (e.g., young, old, male, female), and then these responses could be compared to the characteristics of the person depicted in the original, unaltered, image ${ }^{13}$.

In addition to generating more precise estimates of perceptual versus decisional contributions to MFT performance across groups, the contribution of other impairments needs to be taken into account. For example, there is evidence that attention to a stimulus induces a conservative perceptual bias ${ }^{44}$ and a shift towards stronger evaluation of sensory evidence (and therefore reduced reliance on priors) ${ }^{45}$; such findings raise the possibility that some of the effects we observed could have been due, in part, to less focused attention and subsequently a more liberal and prior-based task strategy among CHR subjects.

While the present data do not allow for strong conclusions to be drawn regarding the causes of the increase in reported face perception in CHR subjects, the findings are intriguing, and they suggest several specific directions for larger follow-up studies. Primary among these, the significantly greater number of reported faces in the CHR group raises the possibility of heightened perceptual sensitivity during the prodromal period of psychosis. As noted above, this has been observed in perceptual organization in high-risk subjects (whereas first episode and later episode schizophrenia patients demonstrate impairments), and in contrast sensitivity in unmedicated first-episode patients (whereas chronically ill patients show an impairment). The significant relationship between greater face reporting and perceptual abnormalities in male CHR subjects is consistent with the data of Teufel et al. and with the hypothesis that perceptual changes and an excessive reliance on priors (i.e., alterations in predictive processing) may be related characteristics of $\mathrm{CHR}$ patients with attenuated psychotic symptoms. This hypothesis was also suggested in a recent paper in which face detection in a task with 40 face-in-noise stimuli and 60 pure noise stimuli was examined in a group of 39 young adults $^{46}$. Results indicated that increased reporting of faces in response to noise stimuli was correlated with scores on the Cardiff Anomalous Perception Scale ${ }^{47}\left(r_{\mathrm{s}}=.50, p=0.001\right)$ and scores on the Peters Delusion Inventory ${ }^{48}\left(r_{\mathrm{s}}=0.44, p=0.005\right)$; the authors suggested that these findings may reflect the influence of overly strong priors for socially meaningful stimuli in ambiguous contexts in people prone to psychosis. In the present study, however, the general lack of strong correlations between MFT scores and symptoms among the CHR participants suggests that it is best to remain agnostic as to MFT-symptom relationships, and the potential for shared mechanisms, until further data are available. The major interpretive issue involving the study, that of perceptual versus decisional contributions to the data, must be considered an open question. Given the unexpectedly (in the context of literature on schizophrenia and CHR groups in general) 'superior' performance of the CHR group, however, further exploration of this issue could be potentially fruitful for risk detection initiatives, especially to the extent that the findings are found to have a perceptual rather than a decisional or motivational basis.

\section{METHODS \\ Participants}

CHR subjects were recruited from specialized CHR clinics at two sites: The Adolescent Development and Preventative Treatment (ADAPT) Program at Northwestern University (PI: Mittal), and the Georgia Psychiatric Risk Evaluation Program (G-PREP) at the University of Georgia (PI: Strauss). Both programs received referrals from local clinicians (e.g., psychiatrists, psychologists, social workers, school psychologists) to perform diagnostic assessment and monitoring evaluations for youth reporting experiencing psychotic-like symptoms, and for youth with a family history of a psychotic disorder who are reporting an increase in general psychopathology symptoms (e.g., depression, anxiety). All CHR participants met criteria for a psychosis-risk syndrome according to the Structured Interview for Psychosis-Risk Syndromes (SIPS) ${ }^{49,50}$. Healthy control (HC) participants were recruited from surrounding communities via online and printed advertisements. Controls did not meet lifetime diagnostic criteria for a DSM-5 psychiatric disorder as determined by the SCID- $5^{51}$, had no family history of psychosis, no lifetime history of neurological disorders, and were not currently prescribed psychotropic medications.

The study was approved by the internal review boards (IRBs) at Northwestern University and the University of Georgia. All participants provided written informed consent, on IRB-approved consent forms, for their involvement in the study.

\section{Mooney faces test}

In the original version of the test ${ }^{19}$, there were 50 degraded (two-tone) black and white images of faces of men, women, and children of various ages. We used 43 of the 44 images in Landsell's $s^{52}$ adaptation of the test. Each image was presented twice, once in an upright orientation and once in an inverted orientation, in a pseudorandom order. It was expected that participants would report perceiving faces in most of the upright images, and in few of the inverted images (consistent with prior studies). The task instructions were simple and presented on a single screen: "Welcome to the experiment! At the beginning of each trial you will see a black and white image. If you believe the image is a face, please press the 1 key on the keyboard. If you do not believe the image is a face, please press the 0 key." The test was implemented in PsychoPy 1.90.1 ${ }^{53,54}$ using Python 2.

\section{Symptom assessment}

Symptoms were assessed using the SIPS ${ }^{49,50}$. To minimize the number of correlational analyses that were undertaken, we limited our examination of MFT-symptom relationships to only the following symptoms: (1) perceptual abnormalities/hallucinations (SIPS item P4), as we anticipated that this positive symptom would be most related to MFT performance if the tendency to report perceiving faces was related to altered predictive coding; (2) grandiose ideas (P3), a positive symptom we expected to be independent of MFT performance, based on ${ }^{36}$; and (3) disorganized communication (P5), given that severity of disorganization has been found to be related to perceptual organization impairment ${ }^{13,15,55}$. We also examined correlations with total scores on the SIPS positive, negative, and disorganized symptom subscales. We predicted that none of the correlations with subscale scores would be statistically significant, given the inclusion of multiple items which we predicted would be weakly related to predictive coding (e.g., grandiosity) and impaired contextual modulation (e.g., impairment in personal hygiene) on the positive and disorganized subscales respectively, and prior findings suggesting that the mechanisms involved in negative symptoms and MFT performance do not overlap.

\section{Data analysis}

The groups were compared using independent-samples t-tests and mixedmodel analyses of variance. Group differences in gender and race were examined using a Pearson chi-square test. Gender differences and the group $x$ gender interaction were examined using ANOVAs. These were explored because in non-clinical samples, it has been observed that males tend to report perceiving more faces than females on the MFT ${ }^{38-40}$ Pearson correlations were calculated both with and without the Hadi 
correction $^{56,57}$ to exclude the influence of outlying values. When $r$ values were similar in both cases, Pearson $r$ values are reported. When the values differed, Spearman's Rho $\left(r_{s}\right)$ values were calculated and are reported. In one case, both Pearson and Spearman values are reported for the sake of comparison. All $\mathrm{p}$ values are two-sided.

\section{Reporting summary}

Further information on research design is available in the Nature Research Reporting Summary linked to this article.

\section{DATA AVAILABILITY}

The data from this study are available from the corresponding author upon reasonable request.

\section{CODE AVAILABILITY}

The code used in this study, to present the Mooney Faces Test (in PsychoPy) and to score the individual subject raw data files (in Visual Basic for Applications), are available from the corresponding author upon reasonable request.

Received: 6 January 2021; Accepted: 19 March 2021; Published online: 17 May 2021

\section{REFERENCES}

1. McGorry, P. D. \& Mei, C. Ultra-high-risk paradigm: lessons learnt and new directions. Evid. Based Ment. Health 21, 131-133 (2018).

2. McHugh, M. J. et al. The Ultra-High-Risk for psychosis groups: Evidence to maintain the status quo. Schizophr. Res 195, 543-548 (2018).

3. Fusar-Poli, P., Sullivan, S. A., Shah, J. L. \& Uhlhaas, P. J. Improving the Detection of Individuals at Clinical Risk for Psychosis in the Community, Primary and Secondary Care: An Integrated Evidence-Based Approach. Front. Psychiatry 10, 774 (2019).

4. Gold, J. M. et al. Enhancing psychosis risk prediction through computational cognitive neuroscience. Schizophr Bull https://doi.org/10.1093/schbul/sbaa091 (2020).

5. Kafadar, E. et al. Modeling perception and behavior in individuals at clinical high risk for psychosis: support for the predictive processing framework. Schizophr Res. https://doi.org/10.1016/j.schres.2020.04.017 (2020).

6. Powers, A. R., Mathys, C. \& Corlett, P. R. Pavlovian conditioning-induced hallucinations result from overweighting of perceptual priors. Science 357, 596-600 (2017).

7. Keane, B. P., Silverstein, S. M., Wang, Y. \& Papathomas, T. V. Reduced depth inversion illusions in schizophrenia are state-specific and occur for multiple object types and viewing conditions. J. Abnorm Psychol. 122, 506-512 (2013).

8. Reed, E. J. et al. Paranoia as a deficit in non-social belief updating. Elife 9, https:// doi.org/10.7554/eLife.56345 (2020).

9. Demmin, D. L., Davis, Q., Roche, M. \& Silverstein, S. M. Electroretinographic anomalies in schizophrenia. J. Abnorm Psychol. 127, 417-428 (2018).

10. Demmin, D. L., Mote, J., Beaudette, D., Thompson, J. \& Silverstein, S. Retinal functioning and reward processing in schizophrenia. Schizophrenia Res. 219, 2533 (2019).

11. Waltz, J. A. \& Gold, J. M. Motivational deficits in schizophrenia and the representation of expected value. Curr. Top. Behav. Neurosci. 27, 375-410 (2016).

12. Dowd, E. C., Frank, M. J., Collins, A., Gold, J. M. \& Barch, D. M. Probabilistic reinforcement learning in patients with schizophrenia: relationships to anhedonia and avolition. Biol. Psychiatry Cogn. Neurosci. Neuroimaging 1, 460-473 (2016).

13. Uhlhaas, P. J., Phillips, W. A., Mitchell, G. \& Silverstein, S. M. Perceptual grouping in disorganized schizophrenia. Psychiatry Res. 145, 105-117 (2006).

14. Silverstein, S. M. et al. Effects of short-term inpatient treatment on sensitivity to a size contrast illusion in first-episode psychosis and multiple-episode schizophrenia. Front Psychol. 4, 466 (2013).

15. Uhlhaas, P. J., Silverstein, S. M., Phillips, W. A. \& Lovell, P. G. Evidence for impaired visual context processing in schizotypy with thought disorder. Schizophr. Res 68, 249-260 (2004).

16. Mittal, V. A., Neumann, C., Saczawa, M. \& Walker, E. F. Longitudinal progression of movement abnormalities in relation to psychotic symptoms in adolescents at high risk of schizophrenia. Arch. Gen. Psychiatry 65, 165-171 (2008).

17. Schneider, U. et al. Reduced binocular depth inversion in schizophrenic patients. Schizophr. Res 53, 101-108 (2002).
18. Balogh, Z., Benedek, G. \& Keri, S. Retinal dysfunctions in schizophrenia. Prog. Neuropsychopharmacol. Biol. Psychiatry 32, 297-300 (2008).

19. Mooney, C. M. Age in the development of closure ability in children. Can. J. Psychol. 11, 216-226 (1957).

20. Rossion, B., Dricot, L., Goebel, R. \& Busigny, T. Holistic face categorization in higher order visual areas of the normal and prosopagnosic brain: toward a nonhierarchical view of face perception. Front Hum. Neurosci. 4, 225 (2011).

21. Cavanagh, P. in Representations of vision: trends and tacit assumptions in vision research. (ed A. Gorea) 295-304. (Cambridge University Press, 1991).

22. Moore, C. \& Cavanagh, P. Recovery of 3D volume from 2-tone images of novel objects. Cognition 67, 45-71 (1998).

23. Van Belle, G., De Graef, P., Verfaillie, K., Rossion, B. \& Lefevre, P. Face inversion impairs holistic perception: evidence from gaze-contingent stimulation. J. Vis. 10, 10 (2010).

24. Kanwisher, N., Tong, F. \& Nakayama, K. The effect of face inversion on the human fusiform face area. Cognition 68, B1-B11 (1998).

25. Silverstein, S. M. \& Keane, B. P. Perceptual organization impairment in schizophrenia and associated brain mechanisms: review of research from 2005 to 2010. Schizophr. Bull. 37, 690-699 (2011).

26. Silverstein, S. M. Visual perception disturbances in schizophrenia: a unified model. Nebr Symp. Motiv 63, 77-132 (2016).

27. Grutzner, $C$. et al. Deficits in high- $(>60 \mathrm{~Hz})$ gamma-band oscillations during visual processing in schizophrenia. Front Hum. Neurosci. 7, 88 (2013).

28. Phillips, W. A. \& Silverstein, S. M. Convergence of biological and psychological perspectives on cognitive coordination in schizophrenia. Behav. Brain Sci. 26, 65-82 (2003). discussion 82-137.

29. Phillips, W. A., Clark, A. \& Silverstein, S. M. On the functions, mechanisms, and malfunctions of intracortical contextual modulation. Neurosci. Biobehav Rev. 52, 1-20 (2015).

30. Brodski-Guerniero, A. et al. Information-theoretic evidence for predictive coding in the face-processing system. J. Neurosci. 37, 8273-8283 (2017).

31. Brodski, A., Paasch, G. F., Helbling, S. \& Wibral, M. The faces of predictive coding. J. Neurosci. 35, 8997-9006 (2015).

32. Corlett, P. R. \& Powers, A. R. Conditioned hallucinations: historic insights and future directions. World Psychiatry.: Off. J. World Psychiatr. Assoc. 17, 361-362 (2018).

33. Alderson-Day, B. et al. Distinct processing of ambiguous speech in people with non-clinical auditory verbal hallucinations. Brain 140, 2475-2489 (2017).

34. Corlett, P. R. et al. Hallucinations and strong priors. Trends Cogn. Sci. 23, 114-127 (2019).

35. Parnas, J. et al. Visual binding abilities in the initial and advanced stages of schizophrenia. Acta Psychiatr. Scand. 103, 171-180 (2001).

36. Teufel, $C$. et al. Shift toward prior knowledge confers a perceptual advantage in early psychosis and psychosis-prone healthy individuals. Proc. Natl Acad. Sci. USA 112, 13401-13406 (2015).

37. Sun, L. et al. Evidence for dysregulated high-frequency oscillations during sensory processing in medication-naive, first episode schizophrenia. Schizophr. Res 150, 519-525 (2013).

38. Foreman, N. Correlates of performance on the Gollin and Mooney tests of visual closure. J. Gen. Psychol. 118, 13-20 (1991).

39. Verhallen, R. J. et al. An online version of the Mooney Face Test: phenotypic and genetic associations. Neuropsychologia 63, 19-25 (2014).

40. Vigen, M. P., G, R. \& Embree, L. J. Adults' performance on a measure of visual closure. Percept. Mot. skills 55, 943-952 (1982).

41. Schwiedrzik, C. M., Melloni, L. \& Schurger, A. Mooney face stimuli for visual perception research. PLoS One 13, e0200106 (2018).

42. McKeeff, T. J. \& Tong, F. The timing of perceptual decisions for ambiguous face stimuli in the human ventral visual cortex. Cereb. Cortex 17, 669-678 (2007).

43. Rodriguez, E. et al. Perception's shadow: long-distance synchronization of human brain activity. Nature 397, 430-433 (1999).

44. Rahnev, D. et al. Attention induces conservative subjective biases in visual perception. Nat. Neurosci. 14, 1513-1515 (2011).

45. Kok, P., Rahnev, D., Jehee, J. F., Lau, H. C. \& de Lange, F. P. Attention reverses the effect of prediction in silencing sensory signals. Cereb. Cortex 22, 2197-2206 (2012).

46. Stuke, H., Kress, E., Weilnhammer, V. A., Sterzer, P. \& Schmack, K. Overly strong priors for socially meaningful visual signals in psychosis proneness. BioRxiv, https://doi.org/10.1101/47342 (2018).

47. Bell, V., Halligan, P. W. \& Ellis, H. D. The cardiff anomalous perceptions scale (CAPS): a new validated measure of anomalous perceptual experience. Schizophr. Bull. 32, 366-377 (2006)

48. Peters, E., Joseph, S., Day, S. \& Garety, P. Measuring delusional ideation: the 21item Peters et al. Delusions Inventory (PDI). Schizophr. Bull. 30, 1005-1022 (2004). 
49. Miller, T. J. et al. Prodromal assessment with the structured interview for prodromal syndromes and the scale of prodromal symptoms: predictive validity, interrater reliability, and training to reliability. Schizophr. Bull. 29, 703-715 (2003).

50. Woods, S. W., Walsh, B. C., Powers, A. R. \& T.H., M. in Handbook of Attenuated Psychosis Syndrome Across Cultures: International Perspectives on Early Identification and Intervention (eds. Li H., D. I. Shapiro, \& L. J. Seidman) 85-113 (Springer, 2019)

51. First, M. B., Williams, J. B., Karg, R. S. \& Spitzer, R. L. Structured clinical interview for DSM-5-Research version (SCID-5 for DSM-5, research version; SCID-5-RV). (American Psychiatric Association, 2015).

52. Landsell, $\mathrm{H}$. Effect of extent of temporal lobe ablations on two lateralized deficits. J. Physiol. Behav. 3, 271-273 (1968).

53. Peirce, J. W. PsychoPy-psychophysics software in python. J. Neurosci. Methods 162, 8-13 (2007).

54. Peirce, J. W. Generating stimuli for neuroscience using psychoPy. Front Neuroinform 2, 10 (2008)

55. Knight, R. A. \& Silverstein, S. M. in Origins and development of schizophrenia: Advances in experimental psychopathology (eds. Lenzenweger M. \& Dworkin R. H.) 247-295 (APA Press., 1998).

56. Hadi, A. S. Identifying multiple outliers in multivariate data. J. R. Stat. Soc. Ser. B 54, 761-771 (1992)

57. Hadi, A. S. A modification of a method for the detection of outliers in multivariate samples. J. R. Stat. Soc., Ser. B 56, 393-396 (1994).

\section{ACKNOWLEDGEMENTS}

The work reported in this paper was supported by the New York Fund for Innovation in Research and Scientific Talent (NYFIRST) (Silverstein), and the following National Institute of Mental Health (NIMH) grants: R01 MH120090 (Gold), R01 MH112613 (Ellman), R01 MH120091 (Ellman), R01 MH120092 (Strauss), R01 MH116039 (Strauss/ Mittal), R21 MH119438 (Strauss), R01 MH112545 (Mittal), R01 MH1120088 (Mittal), U01 MH081988 (Walker), R01 MH120090 (Waltz), R01 MH112612 (Schiffman), and R01 MH120089 (Corlett/Woods).

\section{AUTHOR CONTRIBUTIONS}

S.M.S. conceived of the study, wrote scoring programs for the perceptual task data analyzed the study data, and was the primary author of the manuscript. J.L.T. wrote sections of the manuscript, assisted with creating the tables, and edited the manuscript. J.M.G, J.S., and J.A.W. contributed to the data analysis plan, assisted with data interpretation, and edited the paper. T.F.W. assisted with data analyses, created Table 2 and Fig. 2a, b, and edited the manuscript. R.E.Z. and V.A.M. enabled and facilitated recruitment for the study at the Northwestern University site, trained research staff on administration of the MFT, assembled the final data set from that site, including all clinical ratings and demographic information, and assisted with data interpretation. L.M.E. contributed to data analysis plan, assisted with data interpretation, and edited the paper. G.P.S. and J.A.L. enabled and facilitated recruitment for the study at the University of Georgia site, trained research staff on administration of the MFT, assembled the final data set from that site, including all clinical ratings and demographic information, and assisted with data interpretation. $\mathrm{E}$. F.W. contributed the focus on sex differences, assisted with the data analysis plan and data interpretation, and edited the paper. E.K., J.K., A.R.P. and P.R.C. assisted with interpretation of the data, and edited the manuscript. A.R.P. and P.R.C also wrote sections of the original manuscript and are co-senior authors on the paper. D.S. wrote and modified the computer program to administer the MFT for this study and assisted with development of the program to score individual subject data.

\section{COMPETING INTERESTS}

The authors declare no competing interests.

\section{ADDITIONAL INFORMATION}

Supplementary information The online version contains supplementary material available at https://doi.org/10.1038/s41537-021-00156-1.

Correspondence and requests for materials should be addressed to S.M.S.

Reprints and permission information is available at http://www.nature.com/ reprints

Publisher's note Springer Nature remains neutral with regard to jurisdictional claims in published maps and institutional affiliations.

\begin{abstract}
(c) Open Access This article is licensed under a Creative Commons Attribution 4.0 International License, which permits use, sharing, adaptation, distribution and reproduction in any medium or format, as long as you give appropriate credit to the original author(s) and the source, provide a link to the Creative Commons license, and indicate if changes were made. The images or other third party material in this article are included in the article's Creative Commons license, unless indicated otherwise in a credit line to the material. If material is not included in the article's Creative Commons license and your intended use is not permitted by statutory regulation or exceeds the permitted use, you will need to obtain permission directly from the copyright holder. To view a copy of this license, visit http://creativecommons. org/licenses/by/4.0/.
\end{abstract}

C The Author(s) 2021 